\title{
Análisis de los accidentes y las lesiones de los motociclistas en México
}

\author{
Luis David Berrones-Sanz
}

Colegio de Ciencia y Tecnología, Universidad Autónoma de la Ciudad de México, Ciudad de México, México

\section{Resumen}

Objetivo: Analizar el tipo de lesiones, las características y la distribución geográfica de los accidentes ocurridos a motociclistas en México. Método: Se realizó análisis descriptivo y asociación de variables de fuentes de información secundaria de los accidentes $(N=41,881)$, el total de lesionados $(N=13,916)$ y los egresos hospitalarios $(N=9,111)$ ocurridos en motocicleta durante el año 2014. Resultados: De los lesionados, el 76.6\% eran hombres, el 53.74\% del total de las mujeres eran pasajeras, el $55.1 \%$ de las defunciones fueron debidas a traumatismos intracraneales, solo el $16.6 \%$ usaron casco y Ios que no lo portaban tuvieron 2.11 (odds ratio [OR]:2.1; intervalo de confianza del 95\% [IC 95\%]: 1.8-2.4) veces mayor probabilidad de lesión en la cabeza. Respecto a la gravedad del accidente, se encontró que los ocurridos en zonas suburbanas (OR:6.58; IC 95\%: 5.69-7.60), en superficies de rodamiento no pavimentadas (OR:4.13; IC 95\%: 3.04-5.61), bajo consumo de alcohol (OR:1.89; IC 95\%: 1.46-2.44), en periodos nocturnos (OR:2.24; IC 95\%: 1.95-2.57) y durante el fin de semana (OR:1.65; IC 95\%: 1.44-1.90) tienen mayor probabilidad de ser accidentes fatales. Conclusiones: $A$ pesar de los avances en materia de seguridad vial, los accidentes en motocicleta continúan en aumento y la prevalencia del uso casco es baja. Se deben difundir los subgrupos y factores de riesgo, además de fortalecer la normatividad sobre la comercialización y la utilización del equipo de seguridad para reducir las lesiones.

PALABRAS CLAVE: Accidentes viales. Casco. Motocicleta. Seguridad vial. Lesiones.

\begin{abstract}
Objective: To analyze the type of injuries and the characteristics and geographical distribution of road accidents where motorcycles were involved in Mexico. Methods: A descriptive analysis of second-hand information sources was conducted, including the number of accidents $(N=41,881)$, total number of injured people $(N=13,916)$ and medical expenses $(N=9,111)$ associated to motorcycle accidents during 2014. Results: Motorcycles represent $13.14 \%$ of the total number of deaths in road accidents in Mexico, and the Southeast region of Mexico registers the highest proportion of fatal injuries. Of the total number of motorcycles, $1.84 \%$ (95\% confidence interval [Cl]: 1.83-1.86) were involved in a collision. 3.64 (95\% Cl: 3.39-3.89) people died and 105.5 (95\% Cl: 104.1-106.8) were injured in every 10,000. Out of the total number of injuries, $76.6 \%$ were male and $53.74 \%$ were women. $55.1 \%$ of deaths were caused by intracranial trauma. Only $16.6 \%$ wore a helmet at the time of the accident, and those not wearing a helmet had a 2.11 (odds ratio [OR]: 2.1; Cl 95\%: 1.8-2.4) higher chance of head injury. Regarding the severity of the crash, those occurred in suburban areas (OR: 6.58; $\mathrm{Cl} 95 \%: 5.69-7.60)$, in unpaved surfaces (OR: 4.13; Cl 95\%: 3.04-5.61), after low alcohol consumption (OR: 1.89; Cl 95\%: 1.46-2.44), at night (OR: 2.24; Cl 95\%: 1.95-2.57) and on weekends (OR: 1.65; Cl 95\%: 1.44-1.90), had the highest chance of turning into a fatal accident. Conclusions: In spite of the progress made in terms of road safety, motorcycle accidents are still increasing, and the use of a helmet is still proportionally low. More information on these groups and risk factors needs to be available
\end{abstract}

\section{Correspondencia:}

Luis David Berrones-Sanz

Prolongación San Isidro, 151, cubículo E046

Col. San Lorenzo Tezonco, Del. Iztapalapa

C.P. 09790, Ciudad de México, México

Fecha de recepción: 09-08-2016

Fecha de aceptación: 14-07-2017

E-mail: luis.berrones@uacm.edu.mx

DOI:10.24875/GMM.017002812

Gac Med Mex. 2017;153:662-671

Contents available at PubMed

www.gacetamedicademexico.com 
so people are better informed. Also, regulations need improvements regarding the use of security equipment like helmets in order to reduce injuries and fatal accidents.

KEY WORDS: Road accidents. Helmet. Motorcycle. Road safety. Injuries.

\section{Introducción}

Las motocicletas resultan atractivas por varios factores: por recreación, por el prestigio, por los costos asociados al vehículo y el rendimiento del combustible, las bajas emisiones contaminantes o la rapidez de los desplazamientos en ciudades cada vez más congestionadas. Sin embargo, los usuarios de las motocicletas tienen un riesgo particularmente alto de lesiones por colisión ${ }^{1-3}$, por lo que el motociclismo es considerado una actividad peligrosa ${ }^{4}$. Aunque en otros países los accidentes en motocicleta se han reducido drásticamente ${ }^{5}$, en México las lesiones son un problema que incrementa junto con la tasa vehicular.

Para disminuir los daños, los esfuerzos están enfocados en reducir las lesiones craneoencefálicas a través de reglamentos de tránsito, que obligan al uso de los cascos de seguridad; sin embargo, se dejan de lado los modelos de la psicología social, en la que se deben utilizar las creencias y conductas de las personas, predecir su comportamiento e influir en una conducción segura. Para elaborar programas de reducción de lesiones a motociclistas es esencial conocer sus características y diferentes variables sociales, económicas, culturales, espaciales y temporales. Así, en este trabajo se realiza un análisis de los accidentes en motocicleta con el propósito de identificar subgrupos y características de motociclistas con un riesgo particularmente alto de accidentes. Inicialmente se considera el comportamiento del parque vehicular y la distribución geográfica de los accidentes, y posteriormente se analizan bases de datos sobre accidentes, egresos hospitalarios y motociclistas lesionados. Se considera que este análisis permitirá identificar elementos clave para establecer acciones específicas que contribuyan a la evaluación y el seguimiento de políticas públicas referentes al uso de motocicletas.

\section{Método}

Se realizó un análisis secundario del Registro de Vehículos de Motor Registrados en Circulación y los Accidentes de Tránsito Terrestre del Instituto Nacional de Estadística y Geografía (INEGI) ${ }^{6,7}$, junto con la base de datos de lesionados y egresos hospitalarios de la Dirección General de Información en Salud ${ }^{8}$.
La población bajo estudio incluye el total de accidentes en motocicletas $(\mathrm{N}=41,881)$, el total de lesionados $(N=13,916)$ y los egresos hospitalarios $(N=9111)$ durante el año 2014. Inicialmente se utilizó la población, el número de motocicletas registradas y el número de accidentes ocurridos en cada entidad federativa para realizar un análisis geográfico y determinar tanto la densidad de motocicletas como la distribución de accidentes a lo largo del país. Posteriormente se utilizaron todas las variables disponibles: región anatómica de la lesión, referencia temporal, características del accidente, referencia geográfica y características del conductor responsable y del lesionado, entre otras. Con esto se realizó un análisis estadístico que incluyó la descripción, la categorización y la asociación de variables para lo que se utilizaron las pruebas de diferencia de proporciones y de ji al cuadrado. En las variables asociadas se realizó el cálculo de la odds ratio(OR) con su intervalo de confianza del $95 \%$ (95\%lC), y principalmente se utilizó para la comparación el tipo de accidente (fatal, no fatal) o las lesiones en la parte anatómica de la cabeza.

Para el año 2014, en México se reportan poco más de 38 millones de vehículos de motor registrados en circulación; de estos, el $67.18 \%$ corresponde a los automóviles, el $0.91 \%$ a los camiones para pasajeros, el $25.94 \%$ a los camiones y camionetas de carga y el $5.97 \%$ a las motocicletas; estas últimas tienen la proporción más alta de la historia. En la figura 1 se puede observar el comportamiento en el número de vehículos que han tenido los automóviles y las motocicletas desde el año 1980, y cómo las motocicletas han tenido en la última década un crecimiento muy acelerado. Así, del año 2004 al año 2014, la cantidad de motocicletas en el país aumentó 3.38 veces, con una tasa de crecimiento medio anual del $15.92 \%$. Sin embargo, el país no se comporta de forma homogénea; por ejemplo, en el crecimiento absoluto del número de motocicletas en las 32 entidades federativas llama la atención que, en esa misma década, solo el Estado de Coahuila tuvo un comportamiento decreciente (-38.18\%), y del resto, tres Estados aumentaron significativamente su número motocicletas: Oaxaca, un $751.47 \%$; Tlaxcala, un 984.98\%; y el Estado de México, con un crecimiento 


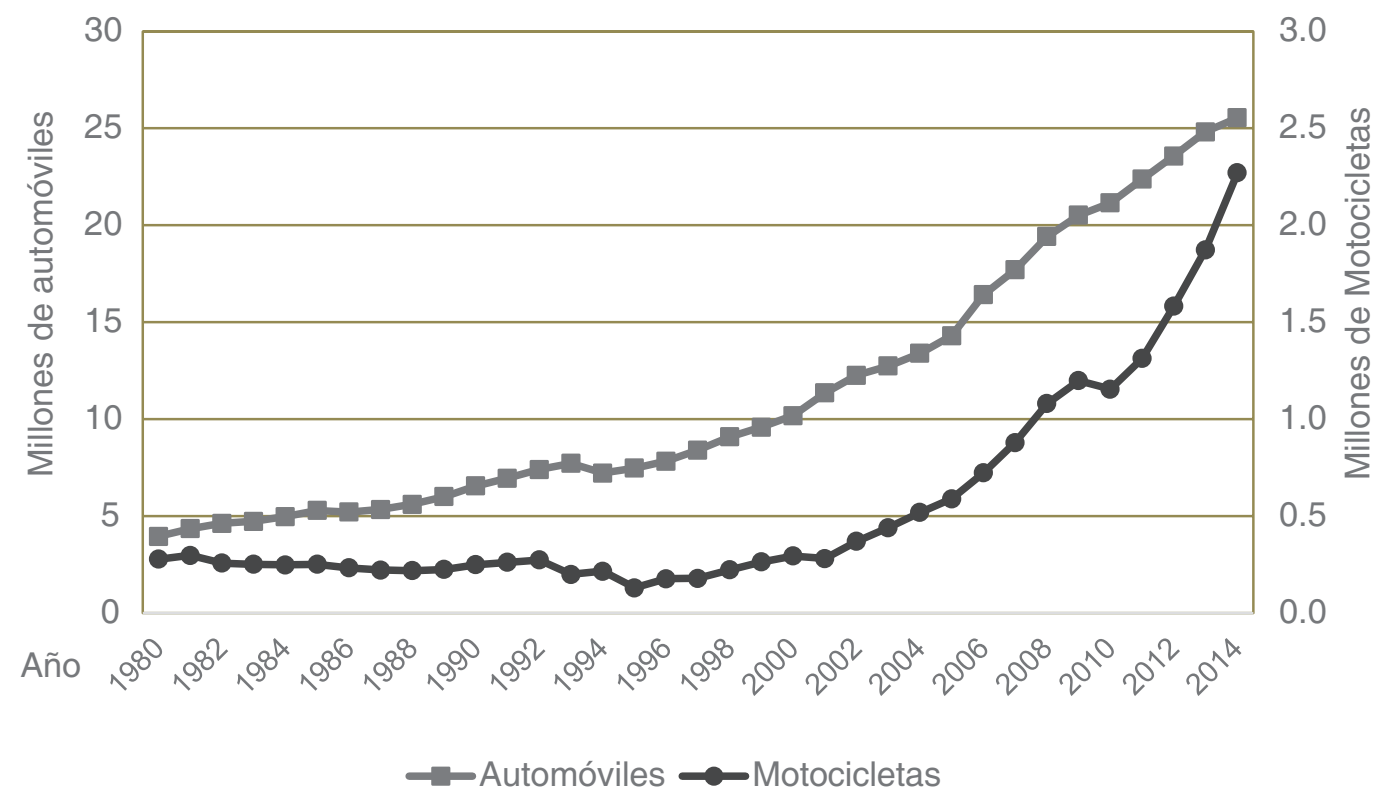

Figura 1. Evolución del parque vehicular en México.

muy superior al promedio, del $4929.01 \%$. No obstante, el Estado de México es la entidad más poblada del país y solo se registran 1.46 motocicletas por cada cien habitantes, por lo que Quintana Roo con un $8.99 \%$, Campeche con un $7.83 \%$ y Yucatán con un $5.82 \%$ son las entidades con más motociclistas por habitantes. Por su parte, Coahuila, con un $0.09 \%$, se mantiene con la menor proporción de motocicletas por habitantes.

Para el año 2014, el INEGI registra en el territorio nacional 41,881 accidentes en motocicleta, por lo que se tienen 34.98 (IC 95\%: 34.6-35.3) accidentes por cada 100,000 habitantes. Como se puede observar en la figura 2, los accidentes tienen una tendencia creciente, y en la última década tuvieron un crecimiento absoluto de un $96 \%$, a un ritmo promedio del $6.95 \%$ por año, en el número de accidentes totales, y un $10.88 \%$ de crecimiento para los accidentes fatales. El aumento en el parque vehicular de las motocicletas se correlaciona positivamente con el número de accidentes $(R=0.93 ; p<0.001)$ y lamentablemente con el número de muertos, cuya tasa pasó de cerca de 2.5a 6.9(IC 95\%: 6.4-7.4) muertos por cada millón de habitantes. Además, las proporciones son más críticas al relacionar los accidentes con el número de vehículos (total de accidentes/total de motocicletas registradas), ya que en el año 2014 el 1.84\% (IC 95\%: 1.83-1.86) de las motocicletas se vieron involucradas en una colisión, murieron 3.64 (IC 95\%: 3.39-3.89) y hubo 105.5 (IC 95\%: 104.1-106.8) lesionados por cada 10,000 motocicletas registradas.
La figura 3 muestra un mapa de la cantidad total de accidentes por entidad federativa; las gráficas de pastel muestran la proporción de colisiones y los puntos señalan la cantidad de motocicletas registradas (parque vehicular) en cada Estado.

El sureste del país (Campeche, Quintana Roo y Yucatán), con la mayor tasa de motocicletas por habitante, coincide con el mayor número de accidentes (108.71, 155.37 y 124.74 por cada 100,000 habitantes, respectivamente), y Quintana Roo es el Estado con la mayor tasa de mortalidad (2.9 muertos por cada 100,000 habitantes) seguido por Sinaloa $(2.4 / 100,000)$ y Aguascalientes $(1.8 / 100,000)$. Resulta interesante que en el norte del país, a pesar de la baja tasa de motorización y de que los accidentes por habitantes se sitúan en valores medios, los accidentes por número de motocicletas registradas se incrementan. Así, en las gráficas de pastel (Fig. 3) se puede observar, por ejemplo, en Coahuila el 51.3\% de las motos registradas sufrieron accidentes, en Sonora el $14.3 \%$ y en Chihuahua el $10.8 \%$. Por el contrario, el Estado de México, a pesar del valor atípico que ha registrado en el incremento de motocicletas, aparece con los valores más bajos de colisiones en todos los indicadores: 4.8 colisiones por 100,000 habitantes y $0.3 \%$ de accidentes en las motocicletas registradas.

En los lesionados por colisión de motocicleta en México predomina el sexo masculino con un $76.6 \%$;de estos, el $49.3 \%$ son menores de 25 años y el $60.1 \%$ 


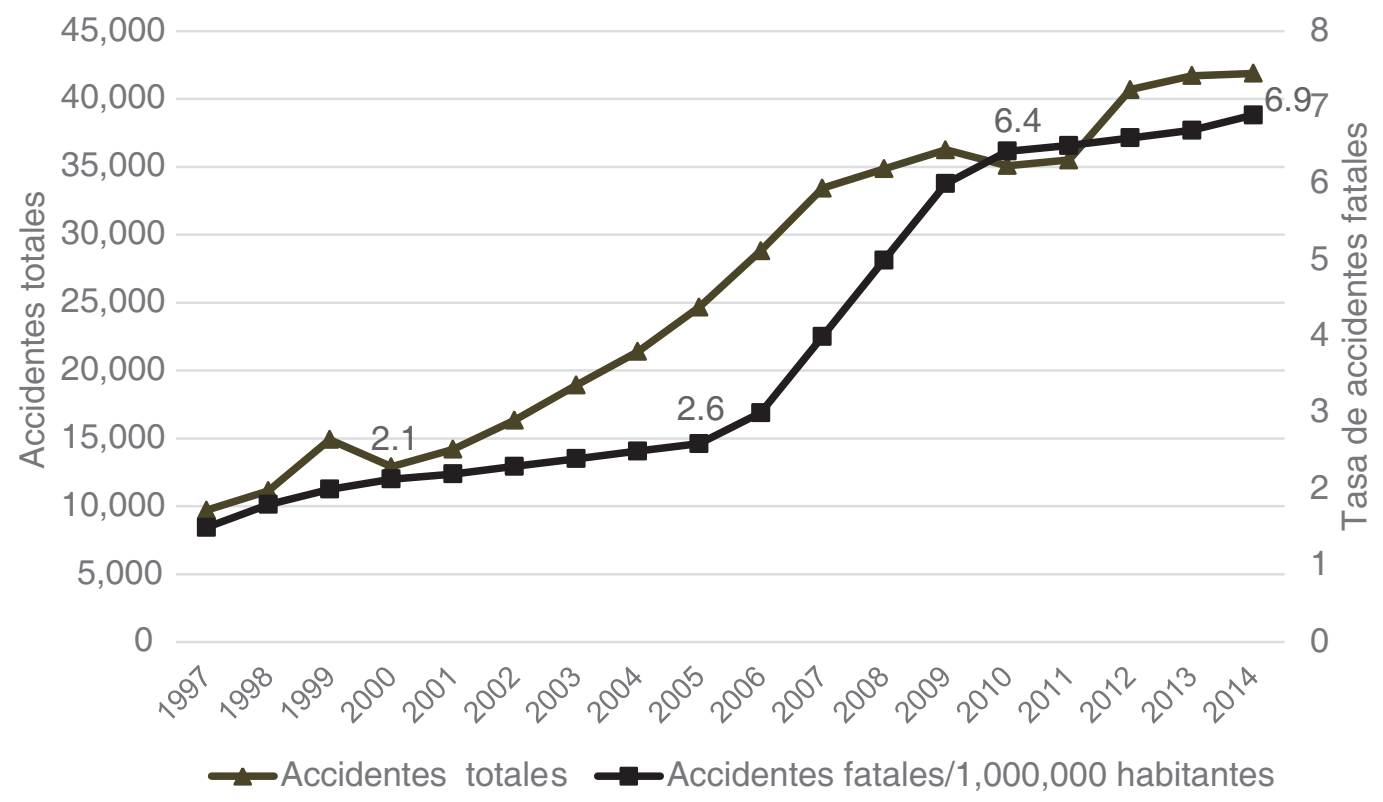

Figura 2. Histórico de colisiones de motocicleta en México.

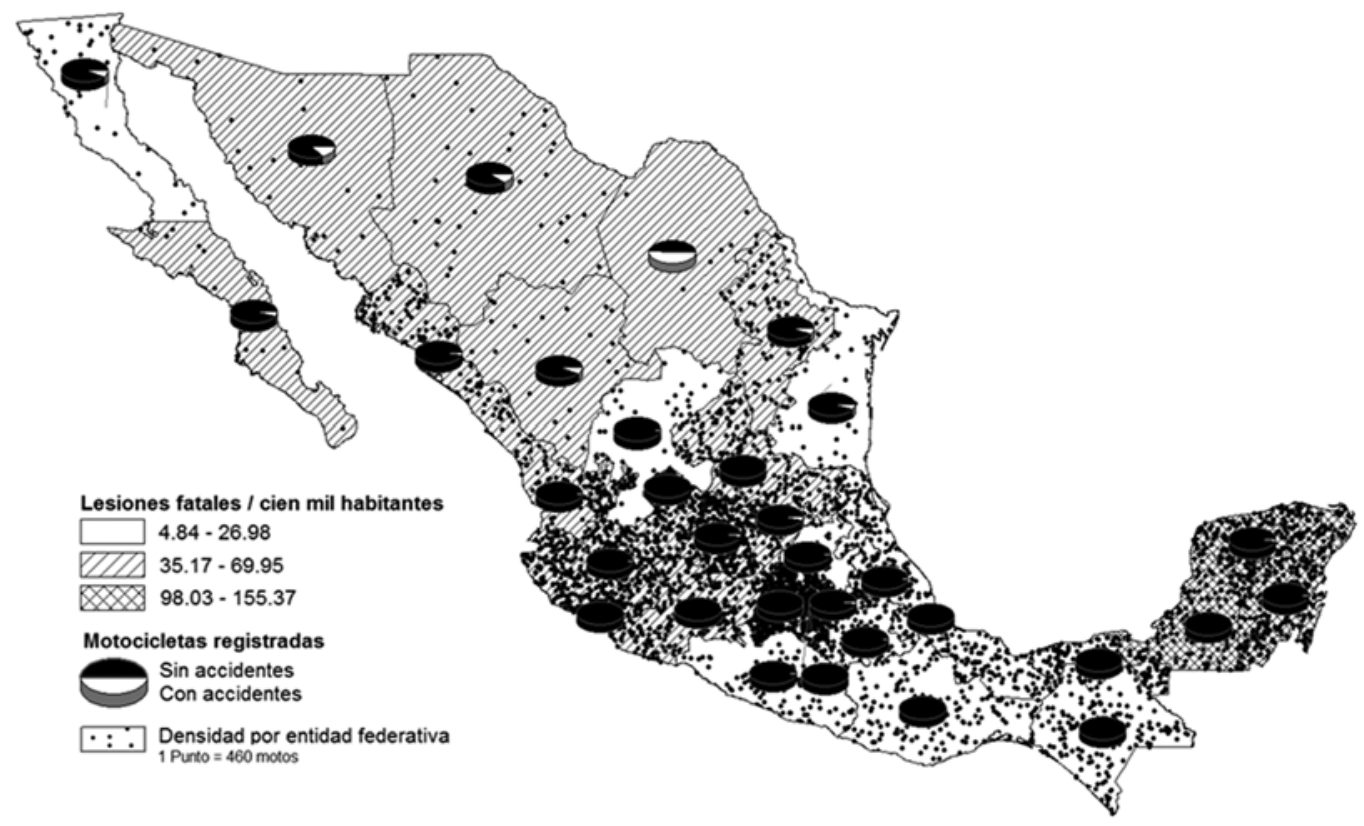

Figura 3. Mapa de lesiones, accidentes y motocicletas registradas. La densidad de motocicletas registradas muestra el parque vehicular correspondiente a la entidad federativa.

tienen 9 años o menos de educación escolar. El sexo representa la principal diferencia entre las características analizadas.

Respecto al tipo de usuario, el $30.2 \%$ de los lesionados no son conductores, sino acompañantes, y en esta categoría las mujeres son las más afectadas; aquí se sitúa el $53.74 \%$ del total del sexo femenino, el mayor porcentaje para este sexo.
Dentro de la temporalidad, el mes de agosto presentó la mayor cantidad de colisiones $(9.73 \%)$ y enero la menor $(6.09 \%)$, principalmente los fines de semana (sábados $15.8 \%$ y domingos $12.7 \%$ ). Otros resultados, como el tipo de accidente, la superficie de rodamiento o la zona donde ocurrió la colisión, que indican que el $98.48 \%$ de los accidentes ocurren en zonas pavimentadas y el $91.76 \%$ en zonas urbanas, se muestran en la tabla 1. 
Gaceta Médica de México.2017;153

Tabla 1. Accidentes de motocicletas según su clase

\begin{tabular}{|c|c|c|c|c|c|c|c|}
\hline \multirow[t]{2}{*}{ Característica } & \multicolumn{2}{|c|}{ Fatal } & \multicolumn{2}{|c|}{ No fatal } & \multicolumn{2}{|c|}{ Solo daños } & \multirow[t]{2}{*}{$p$} \\
\hline & \multicolumn{2}{|c|}{$N=826$} & \multicolumn{2}{|c|}{$\mathrm{N}=23,954$} & \multicolumn{2}{|c|}{$\mathrm{N}=17,101$} & \\
\hline \multicolumn{8}{|l|}{ Sexo del conductor } \\
\hline Se fugó & 162 & $(19.6 \%)$ & 2,995 & $(12.5 \%)$ & 1,722 & $(10.1 \%)$ & $<0.001$ \\
\hline Hombre & 628 & $(76 \%)$ & 18,401 & $(76.8 \%)$ & 13,647 & $(79.8 \%)$ & \\
\hline Mujer & 36 & $(4.4 \%)$ & 2,558 & $(10.7 \%)$ & 1,732 & $(10.1 \%)$ & \\
\hline \multicolumn{8}{|l|}{ Causa del accidente } \\
\hline Conductor & 804 & $(97.3 \%)$ & 22,788 & $(95.1 \%)$ & 16,397 & $(95.9 \%)$ & $<0.001$ \\
\hline Peatón o pasajero & 9 & $(1.1 \%)$ & 390 & $(1.6 \%)$ & 21 & $(0.1 \%)$ & \\
\hline Falla del vehículo & 4 & $(0.5 \%)$ & 90 & $(0.4 \%)$ & 64 & $(0.4 \%)$ & \\
\hline Mala condición del camino & 4 & $(0.5 \%)$ & 359 & $(1.5 \%)$ & 396 & $(2.3 \%)$ & \\
\hline Otra & 5 & $(0.6 \%)$ & 327 & $(1.4 \%)$ & 223 & $(1.3 \%)$ & \\
\hline \multicolumn{8}{|l|}{ Superficie de rodamiento } \\
\hline Pavimentada & 779 & $(94.3 \%)$ & 23,527 & $(98.2 \%)$ & 16,937 & (99\%) & $<0.001$ \\
\hline No pavimentada & 47 & $(5.7 \%)$ & 427 & $(1.8 \%)$ & 164 & $(1 \%)$ & \\
\hline \multicolumn{8}{|l|}{ Zona donde ocurrió el accidente } \\
\hline Urbana & 486 & $(58.8 \%)$ & 21,652 & $(90.4 \%)$ & 16,290 & $(95.3 \%)$ & $<0.001$ \\
\hline Suburbana & 340 & $(41.2 \%)$ & 2,302 & $(9.6 \%)$ & 811 & $(4.7 \%)$ & \\
\hline \multicolumn{8}{|l|}{ Condición de aliento alcohólico } \\
\hline Sí & 74 & $(9 \%)$ & 1,744 & $(7.3 \%)$ & 737 & $(4.3 \%)$ & $<0.001$ \\
\hline No & 338 & $(40.9 \%)$ & 14,920 & $(62.3 \%)$ & 10,247 & $(59.9 \%)$ & \\
\hline Se ignora & 414 & $(50.1 \%)$ & 7,290 & $(30.4 \%)$ & 6,117 & $(35.8 \%)$ & \\
\hline \multicolumn{8}{|l|}{ Edad del accidentado } \\
\hline 0-20 años & 132 & $(16 \%)$ & 3768 & $(15.7 \%)$ & 2701 & $(15.8 \%)$ & $<0.001$ \\
\hline 21-30 años & 223 & $(27 \%)$ & 6530 & $(27.3 \%)$ & 4724 & $(27.6 \%)$ & \\
\hline $31-40$ años & 111 & $(13.4 \%)$ & 4314 & $(18 \%)$ & 3253 & $(19 \%)$ & \\
\hline 41-50 años & 70 & $(8.5 \%)$ & 2653 & $(11.1 \%)$ & 1955 & $(11.4 \%)$ & \\
\hline $51-60$ años & 35 & $(4.2 \%)$ & 1439 & $(6 \%)$ & 1039 & $(6.1 \%)$ & \\
\hline 61-90 años & 11 & $(1.3 \%)$ & 932 & $(3.9 \%)$ & 576 & $(3.4 \%)$ & \\
\hline No especificado & 82 & $(9.9 \%)$ & 1,323 & $(5.5 \%)$ & 1,131 & $(6.6 \%)$ & \\
\hline El conductor se fugó & 162 & $(19.6 \%)$ & 2995 & $(12.5 \%)$ & 1722 & $(10.1 \%)$ & \\
\hline \multicolumn{8}{|l|}{ Día de la semana } \\
\hline Lunes & 108 & $(13.1 \%)$ & 3,359 & $(14 \%)$ & 2,407 & $(14.1 \%)$ & $<0.001$ \\
\hline Martes & 74 & $(9 \%)$ & 3,077 & $(12.8 \%)$ & 2,312 & $(13.5 \%)$ & \\
\hline Miércoles & 92 & $(11.1 \%)$ & 3,181 & $(13.3 \%)$ & 2,422 & $(14.2 \%)$ & \\
\hline Jueves & 98 & $(11.9 \%)$ & 3,027 & $(12.6 \%)$ & 2,368 & $(13.8 \%)$ & \\
\hline Viernes & 88 & $(10.7 \%)$ & 3,560 & $(14.9 \%)$ & 2,719 & $(15.9 \%)$ & \\
\hline Sábado & 172 & $(20.8 \%)$ & 4,113 & $(17.2 \%)$ & 2,709 & $(15.8 \%)$ & \\
\hline Domingo & 194 & $(23.5 \%)$ & 3,637 & $(15.2 \%)$ & 2,164 & $(12.7 \%)$ & \\
\hline \multicolumn{8}{|l|}{ Tipo de accidente } \\
\hline Colisión con vehículo & 48 & $(5.8 \%)$ & 1,562 & $(6.5 \%)$ & 0 & $(0 \%)$ & $<0.001$ \\
\hline Colisión con peatón & 29 & $(3.5 \%)$ & 1,535 & $(6.4 \%)$ & 30 & $(0.2 \%)$ & \\
\hline Colisión con animal & 11 & $(1.3 \%)$ & 107 & $(0.4 \%)$ & 522 & $(3.1 \%)$ & \\
\hline Colisión con objeto fijo & 119 & $(14.4 \%)$ & 838 & $(3.5 \%)$ & 200 & $(1.2 \%)$ & \\
\hline Volcadura & 51 & $(6.2 \%)$ & 851 & $(3.6 \%)$ & 0 & $(0 \%)$ & \\
\hline Caída de pasajero & 14 & $(1.7 \%)$ & 599 & $(2.5 \%)$ & 162 & $(0.9 \%)$ & \\
\hline Salida del camino & 59 & $(7.1 \%)$ & 456 & $(1.9 \%)$ & 4 & $(0 \%)$ & \\
\hline Incendio & 0 & $(0 \%)$ & 7 & $(0 \%)$ & 4 & $(0 \%)$ & \\
\hline Colisión con ferrocarril & 1 & $(0.1 \%)$ & 4 & $(0 \%)$ & 14,558 & $(85.1 \%)$ & \\
\hline Colisión con motocicleta & 461 & $(55.8 \%)$ & 16,864 & $(70.4 \%)$ & 128 & $(0.7 \%)$ & \\
\hline Colisión con ciclista & 12 & $(1.5 \%)$ & 369 & $(1.5 \%)$ & 281 & $(1.6 \%)$ & \\
\hline Otro & 21 & $(2.5 \%)$ & 765 & $(3.2 \%)$ & 0 & $(0 \%)$ & \\
\hline
\end{tabular}


Así, al relacionar el tipo de accidente (fatal o no fatal) con algunas variables se encontró mayor riesgo de morir en las zonas suburbanas (OR:6.58; IC 95\%: 5.69-7.60), las superficies de rodamiento no pavimentadas (OR:4.13; IC 95\%: 3.04-5.61), los horarios nocturnos (OR:2.24; IC 95\%: 1.95-2.57) y los fines de semana (OR:1.65; IC 95\%: 1.44-1.90).

En la tabla 2 se muestran el sexo, la edad, la escolaridad y la región anatómica con lesión de mayor gravedad, clasificadas según el tipo de equipo de seguridad que se utilizó. Las áreas de mayor incidencia de lesiones son las catalogadas como múltiples
(21.76\%), cabeza y cara $(23.15 \%)$, extremidades inferiores $(20.90 \%$ ) y extremidades superiores $(13.60 \%)$, a diferencia de las lesiones que ocasionaron la muerte (Tabla 3), en las que el $55.1 \%$ de las defunciones son atribuidas a traumatismos intracraneales. En general, el $13.73 \%$ se encontraban bajo los efectos del alcohol, con 1.89 (IC 95\%: 1.46-2.44) veces mayor probabilidad de un accidente fatal; un $0.30 \%$ estaban bajo los efectos de drogas ilegales; un $0.17 \%$ consumieron drogas médicas; un $16.6 \%$ de los lesionados traía casco u otro equipo de seguridad; y solo un $9.7 \%$ de los lesionados en la cabeza portaba casco. Así, se

Tabla 2. Características de los motociclistas lesionados según el tipo de equipo de seguridad

\begin{tabular}{|c|c|c|c|c|c|c|c|c|}
\hline \multirow[t]{3}{*}{ Característica } & \multicolumn{8}{|c|}{ Tipo de equipo de seguridad } \\
\hline & \multicolumn{2}{|c|}{ Casco } & \multicolumn{2}{|c|}{ Otro equipo } & \multicolumn{2}{|c|}{ Sin equipo } & \multicolumn{2}{|c|}{ Subtotal } \\
\hline & $N=2310$ & $(100 \%)$ & $N=194$ & $(100 \%)$ & $N=11412$ & $(100 \%)$ & $N=13916$ & $(100 \%)$ \\
\hline \multicolumn{9}{|l|}{ Sexo del lesionado } \\
\hline Masculino & 1813 & $(78.5 \%)$ & 145 & $\left(74.7 \_\%\right)$ & 8,701 & $(76.2 \%)$ & 10,661 & $(76.6 \%)$ \\
\hline Femenino & 497 & $(21.5 \%)$ & 49 & $(25.3 \%)$ & 2,710 & $(23.7 \%)$ & 3,256 & $(23.4 \%)$ \\
\hline No especificado & 0 & $(0.0 \%)$ & 0 & $(0.0 \%)$ & 1 & $(0.0 \%)$ & 1 & $(0.0 \%)$ \\
\hline \multicolumn{9}{|l|}{ Escolaridad } \\
\hline Primaria & 521 & $(22.6 \%)$ & 57 & $(29.4 \%)$ & 2910 & $(25.5 \%)$ & 3489 & $(25.1 \%)$ \\
\hline Secundaria & 885 & $(38.3 \%)$ & 55 & (28.4\%) & 3929 & $(34.4 \%)$ & 4870 & $(35.0 \%)$ \\
\hline Bachillerato & 547 & $(23.7 \%)$ & 42 & $(21.6 \%)$ & 2164 & $(19.0 \%)$ & 2753 & $(19.8 \%)$ \\
\hline Superior & 151 & $(6.5 \%)$ & 16 & $(8.2 \%)$ & 486 & $(4.3 \%)$ & 653 & $(4.7 \%)$ \\
\hline Otra & 92 & $(4.0 \%)$ & 15 & $(7.7 \%)$ & 567 & $(5.0 \%)$ & 674 & (4.8\%) \\
\hline Ninguna & 86 & $(3.7 \%)$ & 6 & $(3.1 \%)$ & 674 & $(5.9 \%)$ & 766 & $(5.5 \%)$ \\
\hline No especificada & 28 & $(1.2 \%)$ & 3 & $(1.5 \%)$ & 682 & $(6.0 \%)$ & 713 & $(5.1 \%)$ \\
\hline \multicolumn{9}{|l|}{ Edad } \\
\hline 0-20 años & 686 & $(29.7 \%)$ & 54 & $(27.8 \%)$ & 4249 & $(37.2 \%)$ & 4989 & $(35.9 \%)$ \\
\hline 21-30 años & 893 & $(38.7 \%)$ & 64 & (33.0\%) & 4089 & $(35.8 \%)$ & 5046 & $(36.3 \%)$ \\
\hline 31-40 años & 396 & $(17.1 \%)$ & 46 & (23.7\%) & 1651 & $(14.5 \%)$ & 2093 & $(15.0 \%)$ \\
\hline 41-50 años & 214 & $(9.3 \%)$ & 18 & $(9.3 \%)$ & 858 & $(7.5 \%)$ & 1090 & $(7.8 \%)$ \\
\hline 51-60 años & 94 & $(4.1 \%)$ & 5 & $(2.6 \%)$ & 359 & $(3.1 \%)$ & 458 & $(3.3 \%)$ \\
\hline 60-99 años & 26 & $(1.1 \%)$ & 7 & $(3.6 \%)$ & 199 & $(1.7 \%)$ & 232 & $(1.7 \%)$ \\
\hline No especificado & 1 & $(0.0 \%)$ & 0 & $(0.0 \%)$ & 7 & $(0.1 \%)$ & 8 & $(0.1 \%)$ \\
\hline \multicolumn{9}{|c|}{ Área anatómica de mayor gravedad } \\
\hline Cabeza & 224 & $(9.7 \%)$ & 42 & $(21.6 \%)$ & 2107 & $(18.5 \%)$ & 2373 & $(17.1 \%)$ \\
\hline Cara & 115 & $(5.0 \%)$ & 10 & $(5.2 \%)$ & 723 & $(6.3 \%)$ & 848 & $(6.1 \%)$ \\
\hline Región ocular & 3 & $(0.1 \%)$ & 0 & $(0.0 \%)$ & 26 & $(0.2 \%)$ & 29 & $(0.2 \%)$ \\
\hline Cuello & 49 & $(2.1 \%)$ & 11 & $(5.7 \%)$ & 214 & $(1.9 \%)$ & 274 & $(2.0 \%)$ \\
\hline Columna vertebral & 16 & $(0.7 \%)$ & 0 & $(0.0 \%)$ & 79 & $(0.7 \%)$ & 95 & $(0.7 \%)$ \\
\hline Extremidades superiores & 344 & $(14.9 \%)$ & 21 & $(10.8 \%)$ & 1527 & $(13.4 \%)$ & 1892 & $(13.6 \%)$ \\
\hline Mano & 89 & $(3.9 \%)$ & 6 & $(3.1 \%)$ & 479 & $(4.2 \%)$ & 574 & $(4.1 \%)$ \\
\hline Tórax & 71 & $(3.1 \%)$ & 13 & $(6.7 \%)$ & 354 & $(3.1 \%)$ & 438 & $(3.1 \%)$ \\
\hline Espalda o glúteos & 21 & $(0.9 \%)$ & 5 & $(2.6 \%)$ & 89 & $(0.8 \%)$ & 115 & $(0.8 \%)$ \\
\hline Abdomen & 14 & $(0.6 \%)$ & 3 & $(1.5 \%)$ & 90 & $(0.8 \%)$ & 107 & $(0.8 \%)$ \\
\hline Pelvis & 23 & $(1.0 \%)$ & 1 & $(0.5 \%)$ & 87 & $(0.8 \%)$ & 111 & $(0.8 \%)$ \\
\hline Región genital & 3 & $(0.1 \%)$ & 1 & $(0.5 \%)$ & 16 & $(0.1 \%)$ & 20 & $(0.1 \%)$ \\
\hline Extremidades inferiores & 600 & $(26.0 \%)$ & 34 & $(17.5 \%)$ & 2274 & $(19.9 \%)$ & 2908 & $(20.9 \%)$ \\
\hline Pies & 101 & $(4.4 \%)$ & 5 & $(2.6 \%)$ & 388 & $(3.4 \%)$ & 494 & $(3.6 \%)$ \\
\hline Múltiples & 577 & $(25.0 \%)$ & 33 & $(17.0 \%)$ & 2418 & $(21.2 \%)$ & 3028 & $(21.8 \%)$ \\
\hline Otros & 54 & $(2.3 \%)$ & 6 & $(3.1 \%)$ & 439 & (3.8\%) & 499 & (3.6\%) \\
\hline Se ignora & 6 & $(0.3 \%)$ & 3 & $(1.5 \%)$ & 102 & $(0.9 \%)$ & 111 & $(0.8 \%)$ \\
\hline
\end{tabular}


encontró que las personas que no utilizan casco tienen 2.11(IC 95\%: 1.82-2.44) veces mayor probabilidad de sufrir una lesión en la cabeza.

\section{Discusión}

Es bien sabido que los motociclistas son un grupo particularmente vulnerable en la carretera. Algunos estudios indican que el riesgo de sufrir lesiones en motocicleta es entre10 y 30 veces superior al correspondiente de los conductores de automóviles ${ }^{5,9}$. Esto se debe no solo a la forma del vehículo, que mantiene el cuerpo a la intemperie y limita, en comparación con los automóviles, las opciones en los sistemas de seguridad pasiva (como el cinturón de seguridad o la misma cabina del vehículo), sino que, además de la falta de protección contra choques, están expuestos a no ser vistos por otros conductores de automóviles y a los impactos con barreras de seguridad que han sido diseñadas para otro tipo de vehículos.

En México, los datos del INEGI indican que las motocicletas contribuyen con un $5.97 \%$ del total de vehículos de motor registrados en circulación, están implicadas en el $5.84 \%$ de los choques y suponen el $13.14 \%$ de los fallecidos por colisiones de tránsito terrestre. Sin embargo, el número de automóviles es 11.25 veces mayor que las motocicletas, y las comparaciones en términos absolutos subestiman la gravedad de las colisiones y la vulnerabilidad de ciertos grupos (que se muestran mejor en términos relativos), ya que tanto el número de vehículos como el de viajes y kilómetros hacen que las cifras de accidentes no sean directamente comparables. A pesar de esto, los accidentes en motocicleta tienen una proporción de 1:10.78 en comparación con los accidentes en automóvil; proporción que cambia a 1:0.96 al considerar, en lugar de la población, la razón de accidentes por el número de vehículos, y a 1:3.5 al considerar solo los accidentes fatales. Sin embargo, el número de motocicletas tiene un crecimiento más acelerado que se correlaciona positivamente con el número de accidentes $(R=0.93 ; p<0.001)$, y las defunciones por esta causa son concordantes con los datos de otras ciudades del mundo, donde han incrementado hasta

Tabla 3. Causas de morbilidad y mortalidad en motociclistas, clasificadas por tipo de egreso hospitalario

\begin{tabular}{|c|c|c|c|c|c|c|}
\hline \multirow[t]{2}{*}{ Causa de morbilidad y mortalidad } & \multicolumn{2}{|c|}{ Otros egresos } & \multicolumn{2}{|c|}{ Defunciones } & \multicolumn{2}{|c|}{ Subtotal } \\
\hline & $N=9,111$ & $(100 \%)$ & $\mathrm{N}=225$ & $(100 \%)$ & $N=9,336$ & $(100 \%)$ \\
\hline Traumatismo intracraneal & 1,199 & $(13.2 \%)$ & 124 & $(55.1 \%)$ & 1,323 & $(14.2 \%)$ \\
\hline Otros traumatismos & 1,879 & $(20.6 \%)$ & 71 & $(31.6 \%)$ & 1,950 & $(20.9 \%)$ \\
\hline Fractura de huesos del cráneo y de la cara & 380 & $(4.2 \%)$ & 4 & $(1.8 \%)$ & 384 & $(4.1 \%)$ \\
\hline Otras fracturas & 483 & $(5.3 \%)$ & 4 & $(1.8 \%)$ & 487 & $(5.2 \%)$ \\
\hline Fractura de la pierna, inclusive del tobillo & 2,114 & $(23.2 \%)$ & 3 & $(1.3 \%)$ & 2,117 & $(22.7 \%)$ \\
\hline Amputaciones de miembros superiores e inferiores & 56 & $(0.6 \%)$ & 3 & $(1.3 \%)$ & 59 & $(0.6 \%)$ \\
\hline Fractura del fémur & 770 & $(8.5 \%)$ & 2 & $(0.9 \%)$ & 772 & $(8.3 \%)$ \\
\hline Heridas de los miembros superiores e inferiores & 235 & $(2.6 \%)$ & 1 & $(0.4 \%)$ & 236 & $(2.5 \%)$ \\
\hline Fracturas de la columna vertebral (cervical, dorsal y lumbar) & 46 & $(0.5 \%)$ & 1 & $(0.4 \%)$ & 47 & $(0.5 \%)$ \\
\hline Fracturas del hombro, del brazo y del antebrazo & 1,096 & $(12.0 \%)$ & 1 & $(0.4 \%)$ & 1,097 & $(11.8 \%)$ \\
\hline $\begin{array}{l}\text { Herida del tórax, del abdomen, de la región lumbosacra y de } \\
\text { la pelvis }\end{array}$ & 29 & $(0.3 \%)$ & 1 & $(0.4 \%)$ & 30 & $(0.3 \%)$ \\
\hline Quemaduras y corrosiones & 13 & $(0.1 \%)$ & 1 & $(0.4 \%)$ & 14 & $(0.1 \%)$ \\
\hline Fractura de la muñeca y de la mano & 193 & $(2.1 \%)$ & 0 & $(0.0 \%)$ & 193 & $(2.1 \%)$ \\
\hline Luxaciones, esguinces y desgarros de regiones múltiples & 333 & $(3.7 \%)$ & 0 & $(0.0 \%)$ & 333 & $(3.6 \%)$ \\
\hline Herida de la cabeza & 117 & $(1.3 \%)$ & 0 & $(0.0 \%)$ & 117 & $(1.3 \%)$ \\
\hline Otras heridas & 58 & $(0.6 \%)$ & 0 & $(0.0 \%)$ & 58 & $(0.6 \%)$ \\
\hline Otras causas & 107 & $(1.2 \%)$ & 8 & $(3.6 \%)$ & 115 & $(1.2 \%)$ \\
\hline Causas mal definidas & 3 & $(0.0 \%)$ & 1 & $(0.4 \%)$ & 4 & $(0.0 \%)$ \\
\hline
\end{tabular}


un $875 \%$ la mortalidad de los motociclistas ${ }^{10}$. Por su parte, se puede inferir que las diferentes proporciones y cantidades atípicas, tanto de motocicletas como de accidentes, en las diferentes regiones del país, son consecuencia de las disparidades en el grado de industrialización, el nivel poblacional, la tasa de motorización y el rápido crecimiento de los moto-taxis como modo de transporte ${ }^{11}$.

Respecto a la parte del cuerpo lesionada, al igual que en otras partes del mundo ${ }^{12-15}$, la cabeza es la más importante; incluso las lesiones catalogadas como heridas múltiples están compuestas en gran medida por lesiones en la cabeza y traumatismos intracraneales. A pesar de los esfuerzos legislativos por normar el uso del casco, la cultura del mexicano es un fuerte componente en las lesiones. Paradójicamente, es común observar que el casco es empleado solo para cumplir con los reglamentos de tránsito, y se utiliza de tamaño o forma inadecuada; por ejemplo, niños con casco de adulto o personas que portan el casco en el brazo. La intención es evitar las sanciones y no proteger de las lesiones. En el reciente reglamento de tránsito de la Ciudad de México ${ }^{16}$, que se presume como uno de los más vanguardistas del país y que entró en vigor en diciembre del año 2015, ya se incorpora, en el artículo 37, que el casco debe ir colocado en la cabeza; sin embargo, no se regulan los cascos, ni en la utilización ni en la comercialización, por lo que es habitual ver cascos muy baratos, pero inadecuados que no cumplen con aspectos de seguridad. Sin embargo, crear normas que obliguen a utilizar cascos seguros, homologados a estándares internacionales, es un tema polémico, principalmente porque los cascos sometidos a estos estándares, como Semell y DOT en los EE.UU. y ECE/ONU R22 en Europa, son dos o tres veces más $\operatorname{costosos}^{17}$. Si sumamos chamarras, guantes, botas y otros equipos protectores, resulta más costoso el equipo de seguridad que una scooter o una de las motocicletas de bajo cilindraje que se comercializan en México. El equipo protector incrementa los costos de la motocicleta y desincentiva este modo de transporte, que ha surgido para suplir las necesidades de movilidad en ciudades con sistemas de transporte público que son incómodos y pocos atractivos para los usuarios. Esto representa un serio problema debido al bajo poder adquisitivo de la población, por lo que a pesar de que la seguridad no debería ser negociable las regulaciones sobre el uso y la comercialización del casco representan medidas no populistas, con costos políticos que los legisladores se resisten a asumir.
Para el caso de México, debido a que las campañas de sensibilización han tenido resultados poco efectivos, se requiere fortalecer la legislación y los mecanismos estrictos que obliguen su cumplimiento, ya que a pesar de que los reglamentos de tránsito, en algunas ciudades, tienen más de una década de haber incorporado el uso del casco como obligatorio, los resultados indican que el $83.99 \%$ de los lesionados no portaban ningún tipo de casco, y esta conducta de riesgo expone a estos motociclistas a 2.11 (IC 95\%: 1.82-2.44) veces mayor probabilidad de sufrir lesiones en la cabeza respecto a los que sí utilizan el casco. Estos resultados coinciden con los de otros estudios que indican que los motociclistas con casco, incluso de cualquier tipo ${ }^{18}$, tienen significativamente menor número de lesiones en la cabeza y el cuello, en todos los niveles de gravedad y para todo tipo de lesiones ${ }^{19}$. Se ha demostrado que un motociclista con casco reduce un $25 \%$ la probabilidad de resultar lesionado y un $30 \%$ si utiliza otro equipo protector (como ropa de cuero), por lo que en conjunto con todo el equipo los riesgos se reducen cerca del $50 \%$.

La prevalencia del uso del casco varía alrededor del mundo; por ejemplo, la literatura muestra valores en países emergentes del $16.2 \%$ en $\operatorname{Laos}^{20}$, el $40 \%$ en Argentina $^{21}$, el $48 \%$ en Camboya ${ }^{22}$ y el $88.7 \%$ en Malasia $^{23}$. Las variaciones se deben a factores culturales, socioeconómicos, ambientales, temporales y normativos propios de cada región ${ }^{24-27}$. Para las ciudades mexicanas, los valores de prevalencia registrados son el $68.9 \%$ en León, el $62.7 \%$ en Guadalajara y el $71.55 \%$ en Cuernavaca, con un promedio del $73.8 \%$ en estas ciudades ${ }^{28}$. No obstante, además de que los resultados son del total nacional y no de un conjunto de ciudades, la cifra de prevalencia del uso del casco en los lesionados es inferior (16.59 vs. $73.8 \%$; $p$ $<0.001$ ); esto se debe a que el estudio de comparación no incluye lesionados, sino puros motociclistas en circulación, y los datos obtenidos son sobre el total de la población lesionada y no sobre motociclistas. Se puede sesgar la muestra debido a que los lesionados pueden asumir mayores riesgos que los motociclistas que no se han accidentado. Sin embargo, existen datos $^{29}$ que indican que al comparar las proporciones parece no haber diferencia; por ejemplo, dentro de los que usan el casco, la mayoría (83.29\%) son conductores y el resto son pasajeros (16.71\%). En el estudio de comparación, esta variable se registra con un $84 \%$, por lo que al realizar la prueba de hipótesis para diferencia de proporciones no puede rechazarse que sean iguales (83.29 vs. $84 \%$; $p=0.520$ ). 
Respecto al uso del casco en los lesionados, en un estudio que se realizó en motociclistas alemanes ${ }^{30}$ se indica que el $72.5 \%$ no usaban casco; cifra más cercana al $83.3 \%$ de los resultados. Sin embargo, el estudio muestra que los motociclistas sufrieron lesiones en la cabeza en proporción del $70 \%$ para los que no usaron casco y del $45 \%$ para los que sí; a diferencia de los resultados donde se obtuvieron proporciones del 18.52 y el $9.70 \%$, respectivamente.

Por su parte, otros factores que se observan en los lesionados son los referentes a la vía y la zona geográfica, donde a pesar de que el $98.48 \%$ de las colisiones ocurren en superficies pavimentadas es 3.3 (IC 95\%: 2.43-4.5) veces más probable un accidente fatal en superficies no pavimentadas y 6.5 (IC 95\%: 5.69-7.60) veces más probable en zonas suburbanas.

Respecto al tipo de accidente, se encontró que el $50 \%$ de las víctimas de la motocicleta implican la colisión con un automóvil, una camioneta u otros accidentes de transporte, y el $42.93 \%$ son accidentes sin colisión. Estas proporciones son similares a las encontradas en otros países ${ }^{31}$, donde alrededor del $60 \%$ de los lesionados y el $40 \%$ de los fallecidos en motocicleta implican la colisión con un coche, y alrededor del $20 \%$ de lesionados y el $25 \%$ de fallecidos son por colisiones de un solo vehículo.

Por otro lado, los días con mayor riesgo son los sábados y domingos, con 1.65 (IC 95\%: 1.44-1.90) veces más probabilidad de accidente que el resto de la semana. Se sabe ${ }^{31}$ que este pico del fin de semana se debe a los paseos recreativos, sobre todo en motocicletas con motores superiores a los 500 centímetros cúbicos, y aunque en México este dato no se tiene disponible, se puede inferir que se comporta de forma similar, debido a que los fines de semana disminuye la utilización de las motocicletas de trabajo que generalmente tienen cilindrajes menores.

En los conductores responsables, mientras que el consumo de alcohol incrementa 1.89 veces el riesgo (IC 95\%: 1.46-2.44), la mayoría (78.02\%) son de sexo masculino, con 2.4 (IC 95\%: 1.70-3.31) veces mayor probabilidad. Esto apoya la creencia de que las mujeres tienen tasas más bajas de participación en los accidentes, en particular entre los conductores jóvenes. Tales diferencias de sexo se han encontrado en los análisis de colisiones de numerosas investigaciones sobre motociclistas ${ }^{23,32}$. Sin embargo, la prevalencia de consumo de alcohol, del $13.73 \%$, es mucho menor que las encontradas en otros estudios ${ }^{33}$ que indican que entre el 41 y el $80 \%$ de los conductores de motocicleta lesionados dieron positivo al consumo de alcohol. Esto puede deberse a un subregistro en la información, pero deberá indagarse al respecto.

Finalmente, a pesar de la importancia de los resultados, es necesario considerar que algunas variables de interés no se incluyeron en este estudio por falta de información; por ejemplo, la base de datos del INEGI no incorpora el equipo de seguridad ni la región anatómica de la lesión, y además existe un gran subregistro en los datos y ninguna de las bases de datos analizadas coinciden en sus cifras, lo que puede deberse a que el formato de atención por violencia o lesión (SIS-SS-17-P) no incluye a la motocicleta como agente de lesión. Otro factor importante es que, durante la construcción de indicadores, las tasas de lesionados y fallecidos no consideran motociclistas o vehículos con varias colisiones. Pese a las limitaciones de la información, los resultados de este estudio forman una aproximación al problema de los motociclistas en México y las variables que se deben considerar durante la elaboración de programas de acción para prevenir sus lesiones. Es evidente que México ha tenido un avance en materia de seguridad vial; por lo menos, desde hace varios años se reconoce como un problema de salud y se han creado iniciativas, organismos y convenios para combatir este problema. El no uso del casco fue uno de los seis factores de riesgo incluidos en el programa de la Iniciativa Mexicana de Seguridad Vial y el uso del casco se ha ido reforzando en la reglamentación del tránsito; además, ya se planean normas para la comercialización de los cascos.

Falta mucho por hacer para lograr reducir las lesiones en los motociclistas, por lo que es importante que se fomenten las buenas prácticas viales, que se promocionen los factores protectores y la utilización de equipos de seguridad para aumentar el porcentaje de personas que utilizan el casco y equipo protector, que se adecue la infraestructura vial a este tipo de vehículos y sensibilizar a los conductores y pasajeros sobre los riesgos de utilizar una motocicleta en, por ejemplo, su uso recreativo, los fines de semana y bajo los efectos del alcohol.

\section{Bibliografía}

1. WHO. Helmets: a road safety manual for decision-makers and practitioners. Geneva: World Health Organization; 2006.

2. Huang B, Preston J. A literature review on motorcycle collisions: final report. Oxford, England: University of Oxford; 2004.

3. Chesham DJ, Rutter DR, Quine L. Motorcycling safety research: a review of the social and behavioural literature. Soc Sci Med. 1993;37:419-29.

4. Mannering FL, Grodsky LL. Statistical analysis of motorcyclists' perceived accident risk. Accid Anal Prev. 1995;27:21-31. 
5. Bjornskau T, Naevestad T-O, Akhtar J. Traffic safety among motorcyclists in Norway: a study of subgroups and risk factors. Accid Anal Prev.2011;49:50-7.

6. INEGI. Vehículos de motor registrados en circulación, 2014. (Accedido el 21 de julio de 2016.) Disponible en: http://www.inegi.org.mx/est/contenidos/proyectos/registros/economicas/vehiculos

7. INEGI. Accidentes de tránsito terrestre en zonas urbanas y suburbanas, 2014. (Accedido el 21 de julio de 2016.) Disponible en: http://www.inegi. org.mx/est/contenidos/proyectos/registros/economicas/accidentes

8. Dirección General de Información en Salud. Sistema Nacional de Información en Salud: Lesiones, 2014.(Accedido el 21 de julio de 2016.) Disponible en: http://www.dgis.salud.gob.mx/contenidos/sinais/estadisticas.htm

9. Elvik R, Vaa T, Monclús J. El manual de medidas de seguridad vial.España: Fundación Instituto Tecnológico para Seguridad del Automóvil; 2006.

10. Silva PHNdV, Lima MLC, Souza WV, et al. Muertes por accidente de motocicleta y su asociación con variables relacionadas a la reproducción social en un estado del noreste brasileño. Salud Colectiva.2015;11:401.

11. Berrones Sanz LD. The working conditions of motorcycle taxi drivers in Tláhvac, Mexico City. Journal of Transport and Health, 2017.

12. Yusuf A, Adeleke N, Adeniran J, et al. Pattern and outcome of motorcyclists head injury in Ilorin, Nigeria. Niger J Basic Clin Sci.2014;11:80.

13. Tosi JD, Ledesma RD, Poó FM, et al. Prevalencia y evolución del uso de casco en ocupantes de motocicletas en una ciudad argentina (Mar del Plata, 2006-2014). SC.2016;12:85.

14. Yu W-Y, Chen C-Y, Chiu W-T, et al. Effectiveness of different types of motorcycle helmets and effects of their improper use on head injuries. Int J Epidemiol. 2011;40:794-803.

15. Oxley J, Ravi MD, Yuen J, et al. Identifying contributing factors to fatal and serious injury motorcycle collisions involving children in Malaysia. Ann Adv Automot Med. 2013;57:329-36.

16. Reglamento de Tránsito del Distrito Federal. Gaceta Oficial del Distrito Federal.2015; 3-122.

17. Ackaah W, Afukaar F, Agyemang W, et al. The use of non-standard motorcycle helmets in low- and middle-income countries: a multicentre study. Inj Prev.2013;19:158-63.

18. Peek-Asa C, McArthur DL, Kraus JF. The prevalence of non-standard helmet use and head injuries among motorcycle riders. Accid Anal Prev. 1999;31:229-33.

19. Hurt HH, Ouellet JV, Thom DR. Motorcycle accident cause factors and identification of countermeasures: volume I: final report.Washington, D.C. Springfield, Va.: National Highway Traffic Safety Administration; 1981.
20. Fong MC, Measelle JR, Dwyer JL, et al. Rates of motorcycle helmet use and reasons for non-use among adults and children in Luang Prabang, Lao People's Democratic Republic. BMC Public Health.2015;15:970.

21. Ledesma RD, Peltzer RI. Helmet use among motorcyclists: observational study in the city of Mar del Plata, Argentina. Rev Saúde Pública 2008;42:143-5.

22. Bachani AM, Branching C, Ear C, et al. Trends in prevalence, knowledge, attitudes, and practices of helmet use in Cambodia: results from a two year study. Injury. 2013;44:S31-7.

23. Ramli R, Oxley J, Hillard $\mathrm{P}$, et al. The effect of motorcycle helmet type, components and fixation status on facial injury in Klang Valley, Malaysia: a case control study. BMC Emerg Med. 2014;14:17.

24. Xuequn $Y, K e L$, Ivers $R$, et al. Prevalence rates of helmet use among motorcycle riders in a developed region in China. Accid Anal Prev. 2011;43:214-9.

25. Zamani-Alavijeh $F$, Bazargan $M$, Shafiei $A$, et al. The frequency and predictors of helmet use among Iranian motorcyclists: aquantitative and qualitative study. Accid Anal Prev. 2011;43:1562-9.

26. Akaateba MA, Amoh-Gyimah R, Yakubu I. A cross-sectional observational study of helmet use among motorcyclists in Wa, Ghana. Accid Anal Prev. 2014;64:18-22.

27. Nicholson SL, Donaghy M, Johnston M, et al. A qualitative theory guided analysis of stroke survivors' perceived barriers and facilitators to physical activity. Disabil Rehabil. 2014;36:1857-68.

28. Lunnen JC, Pérez-Núñez R, Hidalgo-Solórzano E, et al. The prevalence of motorcycle helmet use from serial observations in three Mexican cities. Int J Inj Contr Saf Promot.2014;22:368-76.

29. Hidalgo-Solorzano E, Inclán-Valadez C, Pérez-Núñez R, et al. Motorcycle non-standard helmet use in an urban area of Mexico. Inj Prevent.2012;18:A192.

30. Otte $D$, JessI P, Suren EG. Impact points and resultant injuries to the head of motorcyclists involved in accidents, with and without crash helmets. Proceedings of the 1984 International Conference on the Biomechanics of Impacts. The Netherlands, 4-6th 1984.

31. Elliott MA, Baughan CJ, Broughton J, et al. Motorcycle safety: a scoping study.Transport Research Laboratory; 2003.

32. Mannering FL. Male/female driver characteristics and accident risk: some new evidence. Accid Anal Prev. 1993;25:77-84.

33. Preusser DF, Williams AF, Ulmer RG. Analysis of fatal motorcycle crashes: crash typing. Accid Anal Prev. 1995;27:845-51. 